\title{
REVIEW
}

\section{Root Resorption - A Review}

Kavitha $S^{1}$

ABSTRACT: A thorough knowledge of the aetiological factors and complex dynamics of the mechanisms involved in root resorption are critical for effective management. Poorly diagnosed and treated cases not only have a debilitating physical impact, but also causes psychological trauma to patients. This further stresses on the need for dentists, and endodontists in particular, to be able to recognise cases of established resorption or potential to develop into one. Careful case selection and complete inactivation of resorptive tissues are two critical steps in successful management of root resorption. This review will focus on the pathological process of resorption from a clinician's perspective, and will discuss the various entities of resorption, its features, diagnosis, differential diagnosis, prognosis and treatment. It will also attempt to correlate the aetiopathogenesis to the above mentioned features in order to make the understanding of this obscure pathology clear.

Key words: Root resorption, inflammation, infection, trauma, surface resorption, pressure resorption

Resorption of tooth structure poses challenge to a clinician due to the complexity of the process. The oldest report about resorptions of the dental structures was described by Michael Blum as early as $1530^{[1]}$. Despite the extensive literature on this topic over the decades, it still seems to be poorly understood, due to the inability to correlate the dynamic pathological processes to the clinical scenario and in establishing the treatment guidelines.

Tooth resorption is a complex process involving removal of the organic and inorganic components of tooth structure by clastic cells. It occurs as either a physiological or a pathological process ${ }^{[2]}$. Root resorption of permanent teeth does not occur naturally and is most commonly inflammatory in nature, unlike the physiological resorption that occurs in deciduous teeth. In permanent dentition, it is a pathological event and if untreated, usually results in premature loss of the tooth involved.

By definition, root resorption is a multifactorial process associated with either a physiologic or a pathologic process resulting in dissolution and loss of mineralized tissues like dentin, cementum, as a result of odontoclastic action $^{[3]}$.

\section{Physiological mechanisms inhibiting tooth resorption:}

Several hypotheses have been put forth regarding the resistance of roots to resorption.

1. The remnants of the epithelial root sheath surround the root like a net, imparting a resistance to resorption and subsequent ankylosis.

2. Osteoclasts require a mineralised surface for attachment to initiate resorption. Since the cementoid and predentin that covers cementum and dentine are nonmineralised, osteoclasts adhesion does not take place and this prevents resorption. Furthermore, the cemental layer inhibits movement of toxins from root canal space into the surrounding periodontal tissues.

3. Cementum and PDL cells contain the resorptioninhibition factor, which is a potent collagensae inhibitor. 4. Intermediate cementum or hyaline layer of HopewellSmith, a structureless zone of highly mineralised layer of cementum inhibits the movement of toxins from root canal to the surrounding periodontal tissues.

5. Low molecular weight proteolytic activity inhibitor namely the anti- invasive factor, that is present in cartilage, blood vessels, and teeth, prevent osteoclast attachment and thus the resorption.

\section{Initiation of root resorption:}

Root resorption occurs when there is loss or alteration of 
the protective layer (pre-cementum or pre-dentin). Damage to the protective layer can occur either directly due to the trauma or indirectly when an inflammatory response occurs as a result of a dental injury. The highest possibility of unfavorable outcomes occurs in the case of intrusion, followed by avulsion, whereas the likelihood is least in concussion and subjugation. Inflammation to the unprotected root surface also starts root resorption. Initiation and progression of the inflammation needs continuous presence of the stimulus. This also determines the nature of cells that repopulate the root surface during the phase of healing.

\section{Pathophysiology of root resorption:}

The cells involved in resorption include:

Osteoclasts: These are cells with multi-potential behaviour. They represent a syncytium of stimulated macrophage progenitor cells. The stimulation of these cells is under the control of Receptor activator of nuclear factor kappa B ligand (RANKL)/ Osteoprotegrin (OPG) system $^{[4-13]}$.

Odontoclasts: These are cells that resorb dental hard tissues and are morphologically similar to osteoclasts. Odontoclasts differ from osteoclasts by being smaller in size, fewer nuclei and smaller sealing zones. They have ruffled border with smaller or no clear zones. Osteoclasts and odontoclasts resorb their target tissues in a similar manner.

Macrophages: They are similar to osteoclasts and can become multinucleated giant cells. They lack ruffled border.

\section{Mechanism of resorption by clastic cells ${ }^{[14]}$ :}

Following injury to the predentin and cementoid layer, the actin cytoskeleton of clastic cells attaches to the mineralised tissues, which contain extracellular proteins like osteopontin. This osteopontin molecule serves as a linker between the clastic cell and mineralised tissues. The clastic cell expresses finger like projections called ruffled border, underneath which resorption occurs. The resorptive area is within the clear zone, therefore, is isolated from the extracellular environment, creating an acidic micro environment for the resorption of hard tissue. Thus clastic cells form resorption depressions called Howship's lacunae.

\section{Classification of Root Resorption}

The literature is replete with classifications of root resorption. However, one classification which is most useful from the clinical standpoint is the one by Lindskog et al in 2006. This classification will be followed in this paper.

Table - 1 depicts the Lindskog's Classification $(2006)^{[4]}$

I. Trauma induced tooth resorption

II. Infection induced tooth resorption

III. Hyperplastic invasive tooth resorption

\section{TRAUMAINDUCED RESORPTION}

External surface resorption : This represents the healing response to acute/chronic injury in the PDL. Acute repair-related resorption occurs during luxation injury. Chronic repair-related resorption is normally associated with orthodontic treatment, trauma from occlusion, pressure from developing cysts, apical granuloma and ectopically erupting teeth. These small resorptive lacunae are termed surface resorption, and are usually asymptomatic.

Aetiopathogenesis: The injured tissue adjacent to the root and cementum attract resorbing cells and resorption

\begin{tabular}{|l|l|c|}
\hline \multicolumn{1}{|c|}{ Trauma induced } & \multicolumn{1}{c|}{ Infection induced } & Hyperplastic invasive \\
\hline Surface resorption & Internal inflammatory resorption & Invasive cervical resorption \\
\hline Transient apical resorption & External inflammatory resorption & \\
\hline Pressure resorption & $\begin{array}{l}\text { Communicating internal and } \\
\text { external resorption }\end{array}$ & \\
\hline Replacement resorption & & \\
\hline
\end{tabular}

Table 1 : Classification of Root resorption 
will occur as a part of normal scavenging function of the cells. However the resorbing cells require continuous stimulation for phagocytosis. This type of scavenging involved in initial wound debridement does not appear to be sufficient to sustain the resorption for more than 2 to 3 weeks. In the absence of additional stimulation of the cells, repair with cementum- like tissue occurs. Diagnosis: After 2 or 4 weeks, a localized widening of the PDL space and slight cavitation on the root surface occurs. Most of them cannot be recognized radiographically (Fig-1).

Treatment: Use of mouth props and other protective devices prevent any kind of traumatic injury to the tooth structure and thereby prevents initiation of surface resorption.

Internal Surface Resorption ${ }^{[15,16]}$ : Internal surface resorption is found in areas where re vascularization occurs, such as in fracture lines of root fractures and in apical part of the root canal of a luxated tooth.

Aetiopathogenesis : Osteoclastic activity is a part of the process, along with the formation of granulation tissue. ${ }^{[2]}$

Diagnosis: Transient widening of the root canal is seen in the radiograph.

Treatment: A sign of progressing pulp healing and any endodontic intervention may arrest this process. No treatment is required except periodic observation.

\section{Transient apical internal resorption: ${ }^{[17]}$}

Aetiopathogenesis: This type of resorption normally follows luxation injuries and may be associated with a transient apical breakdown. This is often recognised by a confined periapical radiolucency which resolves within a few months. It is a positive response, with resorptive area allowing ingress of a greater vascular network to aid in the healing of a traumatized pulp.

Diagnosis: Colour change occurs due to intra pulpal hemorrhage and resolves spontaneously if re vascularization to the coronal pulp occurs. As this is a transient process, the internally resorbed apex will close uneventfully.

Treatment: Aesthetic problem may be resolved by external bleaching, but more often elective pulpectomy, root filling and intra coronal bleaching are required. If internal apical resorption is still present, the endodontic treatment should extend only to the position of resorption.

Pressure resorption : The most common type of pressure resorption is orthodontically induced inflammatory root resorption (OIIRR). The inflammation induced by orthodontic force which is essential to the tooth movement, is a fundamental component behind the root resorption process.

Aetiopathogenesis: Intrusive forces of high magnitude, long term orthodontic treatment, treatment started after age 11 with the use of fixed appliances have caused more resorption than with removable appliance. Tooth with blunt or pipette shaped apices are more prone to orthodontically induced root resorption.

There are three degrees of severity of OIIRR:

a. Cemental or surface resorption with remodelling.

b. Dentinal resorption with repair (deep resorption).

c. Circumferential apical root resorption.

Diagnosis: Superficial resorptions normally shows areas of repair and do not result in root shortening. Apical resorptions however result in shortening and blunting of the root tip.

Radiographic appearance: The most common radiographis feature is the "Blunting phenomenon" (rounding of the apex); No radiolucency of bone is notices at the resorption site unlike external inflammatory resorption. In severe cases, crater like appearances are seen ${ }^{[15]}$.

Treatment: It is imperative to cease the orthodontic treatment on identification of resorption. The treatment could be continued with application of light forces and use of NSAIDs. However, the use of NSAIDs in this regard is controversial. Low intensity pulsed ultrasound (LIPUS) may also be used to treat this type of resorption.

Replacement resorption: The most serious form of trauma induced non-infective root resorption is replacement resorption which involves the progressive replacement of tooth structure by alveolar bone and ultimately tooth loss.

Aetiopathogenesis: This type of resorption occurs following death of viable periodontal ligament cells due to compression or drying as in cases of delayed replantation of avulsed teeth ${ }^{[18]}$, inflammatory resorption, and replantation. On rare occasions an intact cementum / cementoid layer may act as a biological barrier, so that ankylosis (i.e. union with bone ) is not accompanied by replacement resorption.

Diagnosis: There is total loss of mobility due to the union of tooth and bone and the tooth gives a high percussion sound, lack of mesial drift, but otherwise the patients are 
asymptomatic. Loss of mobility occurs when more than $10 \%$ of the root surface is ankylosed. The Miller Index, Muhlemann's Macro Periodontometer, Periotest-an electronic device to test the reaction of periodontium to a percussive force and recently, Digital Sound Wave analysis which uses a sound energy are few to diagnose ankylosis. At least $20 \%$ of the root surface must be attached to the adjacent bone for the characteristic percussion sound to be detected. The simplest diagnostic test using percussion with dental mouth mirror is highly specific and sensitive for the diagnosis of ankylosis. (Fig-2).

Radiographic examination: As ankylosis in the initial stages is often on the labial and lingual root surfaces it complicates radiographic detection. There will be total loss of PDL followed by evidence of the progressive replacement of tooth structure by bone giving a moth eaten appearance ${ }^{[19]}$. It is diagnosed radiographically within 2 months after injury.

Treatment: Ankylosis in a developing dentition can severely disrupt arch formation. It fails to erupt and gradually go into infra position. In limited replacement resorption, a surgical repositioning procedure to restore arch integrity is supplemented with application of Emdogain. Emdogain contains enamel matrix proteins or bioactive molecules called amelogenin proteins. These bioactive molecules stimulate tooth development, thus a new cementum, PDL, alveolar periodontal bone is formed. In advanced replacement resorption, a decoronation and submergence procedure ${ }^{[20]}$ allows ongoing alveolar growth both vertically and axially, thus maintaining the labiolingual content of the alveolar process and thus allows reformation of the interdental bone fibers and periosteum. Other treatment approaches are luxation of tooth (breaking of ankylosis sites), vertical distraction, prosthetic elongation and acceptance of the resorbing tooth in slower rate of replacement resorption.

\section{INFECTION INDUCED RESORPTION}

The response of the dento-alveolar apparatus to infection is characterized by inflammation which may result in tooth resorption. This may be a consequence of infective endodontic pathosis alone or can be superimposed on trauma induced resorption. It can be internal or external inflammatory resorption. Internal inflammatory resorption may be classified according to location as

a. Apical, b. Radicular
Apical inflammatory resorption: This type is more common in teeth with various inflammatory periapical pathoses ${ }^{[21]}$.

Radiographic findings: It is difficult to diagnose when the resorption are of the lower grades.

Treatment: Instrumentation should be extended to the position of the resorption with the expectation that with the removal of the micro-organisms followed by the root canal filling hard tissue repair would occur up to the terminus ${ }^{[22]}$. Once repair has occurred, the canals can be filled with gutta-percha and Pro Root MTA can be used as there is increasing evidence for its efficacy when used in similar resorptions ${ }^{[23]}$.

\section{Intra radicular inflammatory internal resorption:}

Internal resorption fully contained within an otherwise intact root will be referred to as intra radicular internal inflammatory resorption. Bell first reported on internal resorption in 1830 . Fothergill referred to the condition as 'pink spot'. In 1920, Mummery published the first extensive study of pink spots and Pritchard showed histologically that internal resorption is comparable to a granuloma of the pulp ${ }^{[24,25]}$. Munch in 1937 was the first to point out that internal resorption can often be delineated radiographically.

Aetiopathogenesis: Inflammation is initiated within the pulp chamber or root canal of the tooth and subsequent resorption can result from trauma, partial pulp removal, caries, pulp capping with $\mathrm{Ca}(\mathrm{OH}) 2$, cracked tooth , extreme heat produced when cutting dentin without an adequate water spray, orthodontics, and anachoresis. Strong medicaments like formocresol could burn the predentine predisposing the root canal to resorption. Herpes Zoster has also been linked with internal resorption. The zoster virus lies dormant for years in the nerve ganglion from an earlier attack of chicken pox and is suddenly reactivated to infect the pulp. A common finding is a large accessory canal communicating from the periodontal ligament to the resorbed area; this may have allowed the passage of a collateral blood supply which probably played an important role in the development and maintenance of internal resorptive process. A vital tissue is required for resorption. The resorption site represents resorbing granulation tissue interposed between healthy and diseased pulp tissue.

Diagnosis: Usually asymptomatic, either positive or negative response. Pink granulation tissue in the coronal dentine is visible undermining the crown enamel. 
Radiographic findings: Intra radicular inflammatory resorption is typically characterized by an oval or round shaped radiolucency enlarging the root canal space within the tooth resorptive process. Fracture of the root will be visible in extensive cases. (Fig-3)

Treatment: Copious irrigation with $5.25 \%$ sodium hypochlorite is mandatory during root canal treatment. This serves to loosen and dissolve the attached pulpal remnants in inaccessible areas, thereby removing all resorptive tissue. The process of irrigation should be augmented by ultrasonic activation coupled with high volume flushing. This is is effective is dissolving all pulp tissue. An interim calcium hydroxide dressing can be placed. Recent evidence shows that Mineral Trioxide Aggregate is a preferred treatment for sealing ${ }^{[26]}$. Obturation of root canals with internal resorption is done using vertical compaction of warm gutta-percha, along with thermoplasticized guttapercha or pressure syringe injection to obtain a three dimensional obturation (Fig4). Greater compaction pressure is required if the defect is large. But this must be avoided in which a perforation or near perforation of the tooth wall exists ${ }^{[27]}$. The use of fibre posts enhances the fracture resistance of these teeth, by distributing functional forces through the tooth's long $\operatorname{axis}^{[26]}$.

\section{External Inflammatory Resorption}

Aetiopathogenesis : This type of resorption occurs when infection is superimposed on a traumatic injury, usually following replantation of an avulsed tooth or a luxation injury. Bacteria primarily located in the pulp space and in dentinal tubules, or in perio-sulcus triggers a macrophage and osteoclast attack on the root surface. Osteoclasts are exposed to toxins from bacteria such as lipopolysaccharide, murmyl dipeptide, and lipoteichoic acid. This results in continuation of the osteoclastic process leading to resorption of the adjacent alveolar bone as these toxins are direct activators of osteoclastic activity. This type of resorption can affect all parts of the root and is typically diagnosed 2-4 weeks after injury. The resorption is a rapidly progressing process that may result in total resorption of the root within few months.

It is more severe in apical third than in the middle and cervical third of root canal because:

1. Inflammation is confined to a very small area at the root apex producing a very high concentration of resorptive factors enough to overcome the resistance of the cemental layer to resorption.
2. Junction of cementum and dentine at the apical foramen provides an extremely thin protective layer compared to the other areas of the tooth.

Diagnosis: As it is chronic in nature it is generally asymptomatic unless the infection becomes acute. Tooth will have increased mobility, dull percussion tone and may be extruded with an overlying swelling or sinus tract. Pulp testing gives no response.

Radiographic Findings: The defect appears as progressive cavitation involving the root and adjacent alveolar bone. It is recognized as bowl like radiolucency. If resorption is extensive there might be loss in normal apical root shape or total loss of root structure (Fig-5).

Treatment: The management strategy varies from a mature and immature tooth. In a mature tooth, the following treatment is provided:

The most important consideration is the thorough debridement of the root canal system. Sequential use of Sodium hypochlorite (3\%), 17\% EDTAC, and a final rinse with EDTAC solution results in a root surface that is free of organic and inorganic debris. This also removes the smear layer thus facilitating the diffusion of medicaments through dentin to the external root surface $^{[28]}$.

The use of a corticosteroid-antibiotic combination (Ledermix paste) as an initial intra canal medicament is known to act as an anticlastic agent. The corticosteroids exerts a positive effect on the clastic cells. Electrophoretically activated copper plus $\mathrm{Ca}(\mathrm{OH}) 2$ has strong antibacterial effect and improved penetration. The antibacterial activity may be enhanced by the use of points containing 5\% chlorhexidine diacetate (Activ Points). Ultimately, during the phase of obturation, obtaining a good apical seal by warm vertical comdensation of gutta-percha is preferred.

In an immature tooth, the following technique may be followed:

Apexification procedures using calcium hydroxide takes many months to obtain enough apical barrier but long term use (more than 30 days) dissolves the organic component of dentin resulting in cervical root fracture. By using MTA as a physical barrier apically, a root canal filling can be placed immediately without waiting for a biological response.

Table : 2 depicts the differential diagnosis of internal, external resorption ${ }^{[29,30]}$. 


\section{Communicating external \& internal resorption: In} this the resorption has extended from an internal inflammatory resorption to involve the external surface, thus a communicating lesion is created.

Clinical findings: Even after apparent removal of all pulp tissue, there is continued hemorrhage in the canal. There is also an additional problem of periodontal inflammation and bleeding due to the communication. In some cases of long standing perforation, a sinus tract may be evident adjacent to the defect.

Radiographic findings: Not diagnosed radiographically unless there is a lateral radicular lesion adjacent to the resorptive defect. In some cases, radiolucency within the tooth structure extending to the exterior surface and the surrounding bone will be visible.

Treatment: The treatment approaches can be nonsurgical or surgical.

Non-surgical repair: Induce calcification at / or within the resorptive lesion by placing medicaments to stimulate periodontal repair and osseous tissue regeneration. Topical application of $90 \%$ aqueous trichloroacetic acid to resorptive tissue for 1 or $2 \mathrm{~min}$ induces coagulation necrosis of the resoptive tissue thus acting as a nidus for calcification - a process called as scaffolding.

Surgical repair: Full mucoperiosteal flap is elevated, the defect curetted, cleaned and restored with GIC / MTA, after the root canal is temporarily obturated with GP cones to avoid blocking of the canal. For defects that are localised in the apical third of the root, surgical endodontic treatment followed by root end resection is performed. In multi-rooted teeth, root resection is the treatment of choice. If the perforation resorption is in an inaccessible area, if no other treatment option is feasible, intentional re-implantation should be considered.

\section{HYPERPLASTIC INVASIVE RESORPTION}

This group of resorptions are insidious in nature and generally present complex therapeutic challenges. These hyperplastic invasive resorptions may have a pulpal (internal) or periodontal origin. The resorbing tissue invades the hard tissues of the tooth in a destructive and apparently uncontrolled fashion ${ }^{[32]}$. Hyperplastic resorptions may be subdivided into

1. Internal replacement resorption

2. Invasive coronal resorption

3. Invasive cervical resorption

4. Invasive radicular resorption.
The most common type of hyperplastic invasive resorption is invasive cervical resorption, otherwise known as external cervical resorption as described by Heithersay. Other terms used to describe invasive cervical resorption includes odontoclastoma, peripheral cervical resorption, extra canal invasive resorption, supra-osseous extra canal invasive resorption, peripheral inflammatory root resorption and sub-epithelial external root resorption

Invasive cervical resorption: It is a clinical term used to describe a relatively uncommon, insidious and often aggressive form of external tooth resorption, which may occur in any tooth in the permanent dentition. It is characterized by its cervical location and invasive nature. This resorptive process leads to progressive and usually destructive loss of tooth structure.

Aetiopathogenesis: Invasive cervical resorption is regarded as a purely an inflammatory reaction. It has been described as an aseptic resorptive process ${ }^{[33]}$, which may sometimes become secondarily invaded with microrganisms. The microrganisms from either the gingival sulcus or the pulp space or the dentinal tubules in teeth with necrotic pulps provide the necessary stimuli to sustain these invasive cervical resorptive lesions. ${ }^{[34]}$ Certain factors that can initiate the resorption are orthodontic treatment, trauma, intracoronal bleaching and periodontal therapy ${ }^{[33]}$. Other predisposing factors include bruxism, intracoronal restorations, and developmental defects such as hypoplasia or hypomineralization of cementum. Systemic diseases such as hyperoxaluria and oxalosis cause an increased concentration of oxalates in blood. In association with kidney failure, there is further precipitation of crystals in hard tissues which could initiate the root resorptive process $^{[35]}$.

Classification: Invasive Cervical resorption is classified as follows :

1. Franks classification (Fig-6)

2. Heithersay classification: (Based on the severity). (Fig-7)

Class 1-A small invasive resorptive lesion is present near the cervical area with shallow penetration into dentine. Class 2- A well-defined invasive resorptive lesion that has penetrated close to the coronal pulp chamber but shows little or no extension into the radicular dentine. Class 3-A deeper invasion of dentine by resorbing tissue, not only involving the coronal dentine but also extending 


\begin{tabular}{|c|c|c|}
\hline & External & Internal \\
\hline \multirow{2}{*}{$\begin{array}{c}\text { APICAL THIRD } \\
\text { Common feature: Blunted/ } \\
\text { shortened apex }{ }^{[31]}\end{array}$} & $\begin{array}{l}\text { Canal size smaller and walls } \\
\text { converge apically }\end{array}$ & $\begin{array}{l}\text { Incomplete root formation } \\
\text { Canal system is large with } \\
\text { parallel root }\end{array}$ \\
\hline & & $\begin{array}{l}\text { Divergent walls / blunder bus } \\
\text { canal }\end{array}$ \\
\hline \multirow{7}{*}{$\begin{array}{l}\text { MIDDLE THIRD } \\
\text { Common feature: } \\
\text { Radiolucent lesion }\end{array}$} & $\begin{array}{l}\text { Less well defined margins; ragged } \\
\text { and irregular, shows variations in } \\
\text { density that may appear striated. }\end{array}$ & $\begin{array}{l}\text { Margin will be sharp, smooth } \\
\text { and are clearly defined. }\end{array}$ \\
\hline & Moth eaten appearance & Uniform in density \\
\hline & Not symmetrical & Mostly symmetrical \\
\hline & $\begin{array}{l}\text { Lesion may be superimposed over } \\
\text { the canal outline }\end{array}$ & $\begin{array}{l}\text { Canal or chamber appears to } \\
\text { balloon out }\end{array}$ \\
\hline & Canal unaltered till the apex & $\begin{array}{l}\text { Canal not present in the area of } \\
\text { lesion }\end{array}$ \\
\hline & $\begin{array}{l}\text { Lesion shifts from its } \\
\text { superimposed position during } \\
\text { angulated radiograph. }\end{array}$ & $\begin{array}{l}\text { Lesion will not shift no matter } \\
\text { how severe the angulation is }\end{array}$ \\
\hline & $\begin{array}{l}\text { If the lesion is located on the } \\
\text { palatal aspect the mesially } \\
\text { angulated radiograph will move } \\
\text { the lesion to the mesial aspect. }\end{array}$ & \\
\hline CORONAL THIRD & Can begin within the clinical & Begin below the epithelial \\
\hline Common Feature-Location & crown & $\begin{array}{l}\text { attachment and then invade } \\
\text { occlusally }\end{array}$ \\
\hline
\end{tabular}

Table 2 : The differential diagnosis of internal, external resorption

\begin{tabular}{|l|l|}
\hline \multicolumn{1}{|c|}{ Invasive cervical resorption } & \multicolumn{1}{c|}{ Subgingival caries } \\
\hline $\begin{array}{l}\text { Base of the defect hard, a scrapping sound } \\
\text { when probed,profuse bleeding on } \\
\text { probing,presence of pink spot. }\end{array}$ & $\begin{array}{l}\text { Sticky on probing, does not present with a } \\
\text { pink spot. }\end{array}$ \\
\hline Positive to sensitivity testing & Varies according to involvement of pulp \\
\hline
\end{tabular}

Table 3 : Differential diagnosis of invasive cervical resorption and subgingival caries 


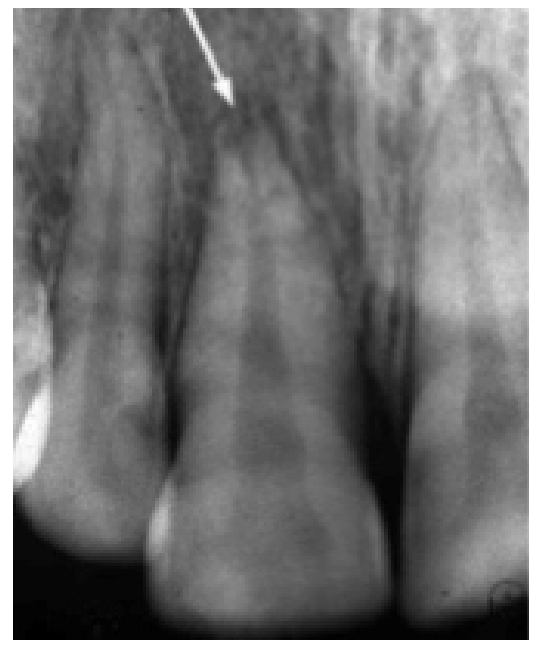

Fig 1: External surface resorption

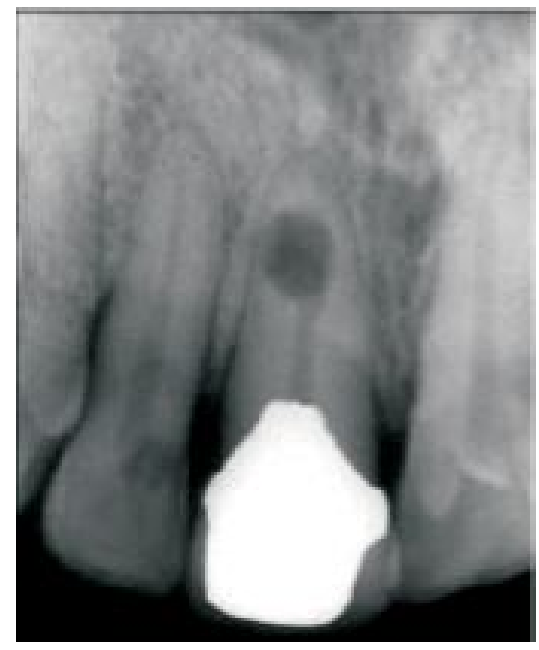

Fig 3: Internal resorption

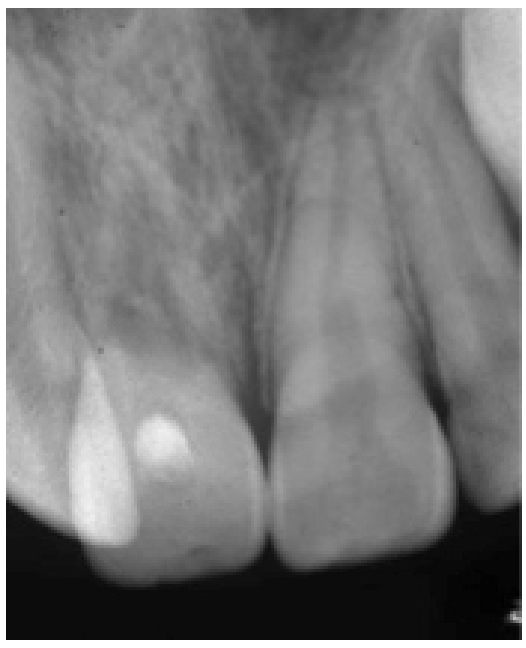

Fig 2: Replacement resorption

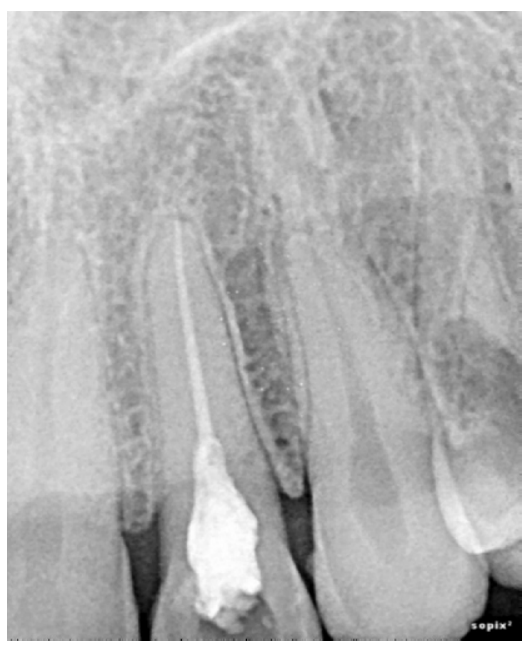

Fig 4: Obturation of tooth with internal resorption

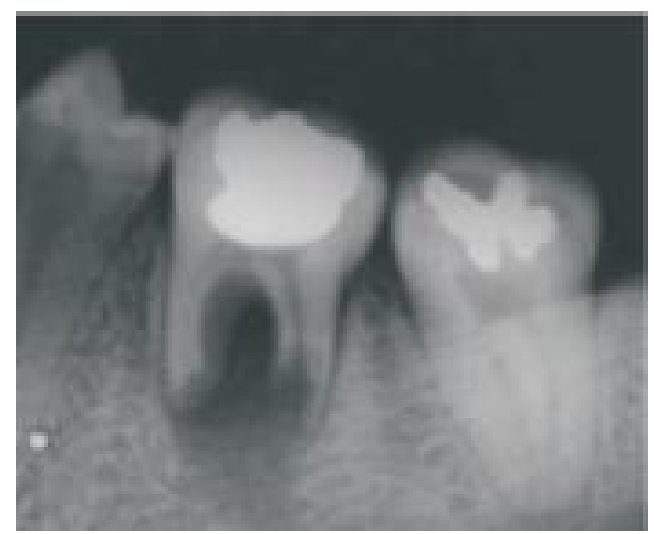

Fig 5: External inflammatory resorption 
into the coronal third of the root.

Class 4-A large invasive resorptive process that has extended beyond the coronal third of the root.

Diagnosis: Diagnosis is based on the following criteria:

1. Lesion will be located in cervical region of tooth.

2. Patient is asymptomatic

3. When resorption is long standing, granulation tissue spreads coronally to undermine the enamel of the crown, resulting in a pinkish appearance, mis diagnosed as internal resorption.

4. Tooth responds positively to vitality tests unless there is pulpal involvement (in very advanced cases).

5. The base of a defect is hard, a scraping sound is heard when probed.

6. Profused bleeding on probing is present due to presence of granulation tissue.

7. The edges of the cavity usually appear sharp.

Table - 3 depicts the differential diagnosis between invasive cervical erosion and subgingival caries.

Radiographic Findings: It varies from asymmetrically located radiolucency with irregular margins in cervical/proximal region of tooth to uniformly round radiolucency centered over the root. They are detected by chance radiologically because tooth is usually asymptomatic. Early lesions are usually radiolucent in appearance and advanced lesions might have mottled appearance because of fibro-osseous nature of the lesion. Radiolucency expands coronally and apically in dentine and reaches the root canal but does not perforate. CBCT is the best option for diagnosing invasive cervical resorption.

Treatment: Treatment depends on the severity, location, whether the defect has perforated the root canal system and the restorability of the tooth. The treatment objectives are to arrest resorptive process, restore damaged root surface, prevent further resorption and improve aesthetics of tooth (in cases where resorption has led to a pink spot). The management can be surgical or non-surgical based on the severity.

Non surgical treatment Class 1, Class 2 type of resorption: Topical application of trichloroacetic acid results in coagulation necrosis of the external cervical resorptive tissue, with no damage to adjacent periodontal tissues. It also infiltrates the small channels and recesses of the defect that would otherwise be unreachable by mechanical instrumentation ${ }^{[30]}$.
Class 3 type of resorption: Topical application of $90 \%$ trichloroacetic acid along with curettage, elective pulpectomy, intracanal dressing, root filling and restoration controls the resorption process. If the margins are subgingivally located, orthodontic extrusion can be done and the exposed defect can be sealed with Mineral Trioxide Aggregate.

Class 4 type of resorption: These defects have to be monitored for prognosis, or be extracted and replaced.

Surgical treatment: The following are the surgical managements that can be done:

1. Periodontal flap reflection and curettage, followed by restoration of the defect.

\section{Apically positioning the flap \\ 3. Guided Tissue Regeneration}

CONCLUSION: As the etiologic factors, diagnosis,
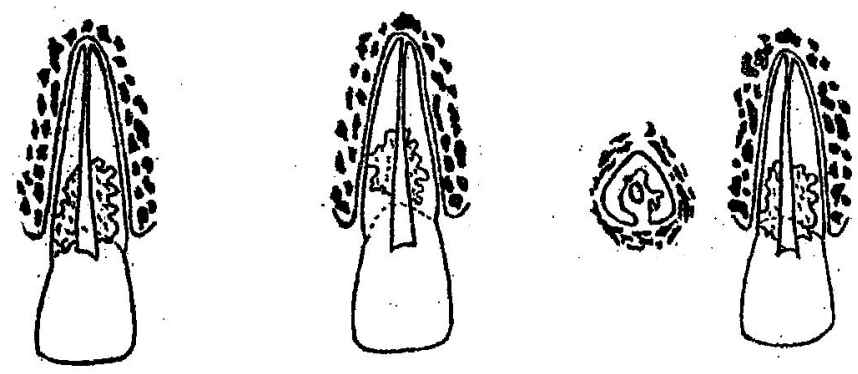

Fig 6: Frank's classification

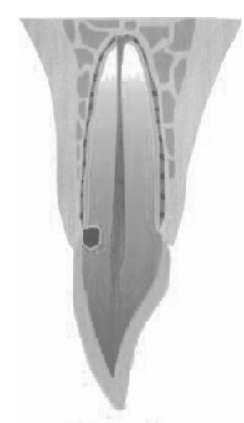

Class 1

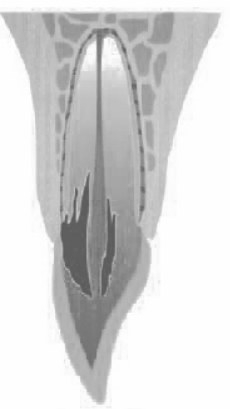

Class 3

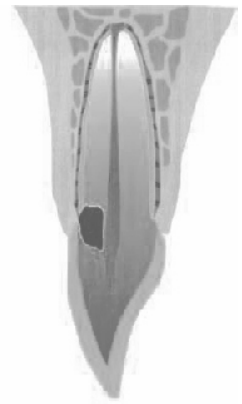

Class 2

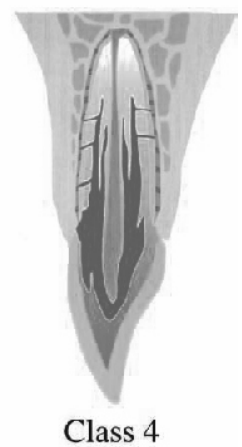

Fig 7: Hiethersay classification of invasive cervical resorption 
treatment and prognosis are different for various types of resorptive defects, the endodontist should be able to diagnose resorption clinically and radiographically and commence appropriate treatment to halt the resorptive processes. A basic understanding of the pathogenesis involved is important to appreciate the importance of meticulous treatment.

\section{REFERENCES :}

1. Silva LB, Guimaraes CS, Santos RA. Immunology of root resorption: a literature review. Indian J Dent Res. 2008;19(4):340-3.

2. Pierce A. Pathophysiological and therapeutic aspects of dentoalveolar resorption. Aus Dent J 1989;34:437- 48

3. Patel S, Pit Ford T. Is resorption external or internal? Dental Update 2007;34:218-29.

4. Heithersay GS. Management of tooth resorption. Aust Dent J 2007;52(1 Suppl):S105-21.

5. Yildrim S, Yapar M, Sermet U, SenerK, Kubar A. The role of dental pulp cells in resorption of deciduous teeth. Oral Surg Oral Med Oral Pathol Oral Radiol Endod 2008;105:113-20.

6. Crotti T, Smith MD, Hirsch R, Sonkoulis S, Weedon $\mathrm{H}$, Capone M. Receptor activator of NF?B ligand (RANKL) and osteoprotegerin (OPG) protein expression in periodontitis. J Periodont Res 2003;38:380-7.

7. Khosla S. Mini review: the OPG/RANKL/RANK System. Endocrinology 2001;142:5050-55.

8. Heinrich J, Bsoul S, Barnes J, Woodruff K, Abboud S. CSF-1,RANKL and OPG regulate osteoclastogenesis during murine tooth eruption. Arch Oral Biol 2000;50:897-908.

9. Wise GE, Ren Y, Yao S. Regulation of osteoprotegerin gene expression in dental follicle cells. J Dent Res 2003;82:298-302.

10. Rani CS, Macdougall M. Dental cells express factors that regulate bone resorption. Mol Cell Boil Res Commun. 2000;3:145-52.

11. Tyrovola JB, Spyropoulos MN, Makou M, Perrea D. Root resorption and the OPG/RANKL/RANK system: a mini review. Oral Sci 2008;50(4):367-76.

12. Le XK, Laflamme C, Rouabhia M. Porphyromonasgingivalis decreases osteoblast proliferation through IL-6-RANKL/OPG and MMP 9/TIMPs pathway. Indian J Dent Res, 2009;20(2):141-49.

13. Lerner UH. Neuropeptidergic regulation of bone resorption and bone formation. Musculoskel Neuron Interact 2002;2:440-47.

14. Andreason JO, Bakland LK. Pathologic tooth resorption. In: Ingle JI, Bakland LK, Baumgartner JC, editors. Ingles Endodontics. 6th ed. BC Decker, Ontario; 2008. pp 135882.

15. Andreasen FM. Transient apical breakdown and its relation to color sensibility changes after luxation injuries to teeth. Endod Dent Traumatol 1986; 2(1):9-19.

16. Arathi R, Kundabala M, Karen B. Disappeared roots: A case report. J Indian Soc Pedod Dent. 2008;26(1):36-39.

17. Malmigram B. Decoronation of an ankylosed tooth for preservation of alveolar bone prior to implant placement. Dent Traumatol 2001;17(2):93-95

18. Vier FV. FIgneirdo JA. Internal apical resorption and its correlation with the type of apical lesion. Int Endod J 2004;37(11): 730-37.

19. Buchannan S. Prosystem GT,design,technique and advantages. Endod Topics 2005;10:168-179.

20. Mummery JH. The pathology of pink spots' on teeth. Br Dent J.1920;41:301-311.

21. Gartner AH, Mack T, Somerlott RG, Walsh LC. Differential diagnosis of internal and external root resorption. JEndod 1976;2(11):329-34.

22. Jacobvitz M, De lima RKP. Treatment of inflammatory internal root resorption with mineral trioxide aggregate: a case report. Int Endod J 2008;41:905-12.

23. Gold berg F, Massone EJ, Esmoris M, Alfie D. Comparison of different techniques for obturating experimental internal resorptive cases. Endod Dent Traumatol 2000; 16:116-121.

24. Ford TR, Torabinejad M, McKendry DJ, Hong CU, Kariyawasam SP.Use of mineral trioxide aggregate for repair of furcal perforations.Oral Surg Oral Med Oral Pathol Oral Radiol Endod. 1995 Jun;79(6):756-63

25. Abbott PV, Heijkoop PS, Cardaci SC, Hume WR, Heithersay GS. An SEM study of the effects of different irrigation sequences and ultrasonics. Int Endod $\mathrm{J}$ 1991;24(6):308-16.

26. Abbott PV, Heithersay GS, Hume WR. The release and diffusion through human coronal dentine in vitro of triamcinolone and demeclocycline from Ledremix Paste.Endod Dental Traumatol 1989;5:92-97.

27. Pierce A, Lindskog S. The effect of an antibiotic/corticosteroid paste on inflammatory root resorption in vivo. Oral Surg Oral Med Oral Pathol 1987;64(2):216-20 
28. Masseler M, Malone AJ. Root resorption in human permanent teeth. A roentgenographic study. Am J Orthod 1954;40:619.

29. Heithersay GS. Clinical, radiologic and histopathologic features of invasive cervical resorption. Quintessence Int 1999;30(1):27-37.

30. Patel S, Kanagasingam S, Pitt Ford T. External cervical resorption:AReview. J Endod 2009;35(5):616- 25.

31. Fuss Z, Tsesis I, Lin S.Root resorption-diagnosis, classification and treatment choices based on stimulation factors. Dent Traumatol2003;19(4):175-82.

32. Moskow BS. Periodontal manifestation of hyperoxalouria and oxalosis. J Periodontol 1989;60(5):271-8.

\section{Address for correspondence:}

Dr. Kavitha S, MDS,

Professor

Department of Conservative Dentistry and Endodontics,

SRM Dental college, Ramapuram,

Chennai - 89.

Email id: kavithakrithya@gmail.com
33. Neely AL, Gordon SC. A familial pattern of multiple idiopathic cervical root resorption in a father and son: a 22 year follow up. J Periodontol 2007;78(2):367-71.

34. Patel S, Dawood A, Whaites E, Pitt ford T. The potential applications of cone beam computed tomography in the management of endodontic problems. Int Endod $\mathrm{J}$ 2007;40(10):818-30.

35. Heithersay GS. Invasive cervical resorption. Endod Topics 2004;7:73-92.

\section{How to cite this article:}

Kavitha S. Root Resorption - A Review. Journal of scientific Dentistry 2012;2(1):12-22

Source of Support: Nil, Conflict of Interest: None declared

\section{Authors:}

${ }^{1}$ Professor

Conservative Dentistry and Endodontics, SRM Dental college, Ramapuram,Chennai 